Voix et Images

\title{
Clopin-clopant dans l'épopée : Le Nouveau Théâtre Expérimental de Montréal
}

\section{Bernard Andrès}

Volume 7, numéro 2, hiver 1982

Michel Tremblay

URI : https://id.erudit.org/iderudit/200328ar

DOI : https://doi.org/10.7202/200328ar

Aller au sommaire du numéro

Éditeur(s)

Les Presses de l'Université du Québec

ISSN

0318-9201 (imprimé)

1705-933X (numérique)

Découvrir la revue

Citer cet article

Andrès, B. (1982). Clopin-clopant dans l'épopée : Le Nouveau Théâtre

Expérimental de Montréal. Voix et Images, 7(2), 397-400.

https://doi.org/10.7202/200328ar d'utilisation que vous pouvez consulter en ligne.

https://apropos.erudit.org/fr/usagers/politique-dutilisation/ 


\title{
Clopin-clopant dans l'épopée : Le Nouveau Théâtre Expérimental de Montréal
}

\author{
par Bernard Andrès
}

Pour les captifs de Montréal au mois d'août, Vie et Mort du Roi boiteux constituait certainement l'événement théâtral estival. Mais avant d'aborder cette «épopée sanglante et grotesque», je dirai deux mots de ce dont j'ai horreur, par nature : le vide. Un mot donc sur d'autres productions réellement grotesques, elles, et qui font vivre toute une industrie : celle des théâtres d'été. Il est vrai que je n'en parle jamais, ou que je n'en parle plus depuis une certaine saison où je me les suis infligés presque systématiquement dans le seul but un peu maso de m'en faire une idée ${ }^{1}$. Mais comment se faire une idée de rien... ou de quelque chose de quasiment imperméable à l'idée d'idée? A Devos de trancher. Ceci dit, peut-être aije manqué à mon devoir de chroniqueur, cet été, en me dispensant d'assister à tel ou tel de ces chefs-d'œuvre de la dramaturgie aoûtienne qui présentent au moins les vertus d'assurer un plein emploi à l'Union des Artistes, de fausser les statistiques de la Main-d'œuvre et d'économiser sur les prestations d'assurance-chômage et les subventions gouvernementales aux Arts d'interprétation... tout en reproduisant la force de travail du citoyen-spectateur en le dotant d'une bonne dose d'humour (?) pour affronter les farces (plates) de la rentree: records au hit parade de l'inflation, du chômage et des taux d'intérêt! Qu'on en juge plutôt sur une simple liste des titres:

Un show d'été pour payer mon loyer,

Les extravagantes et voluptueuses aventures du Baron de Crac,

Les folleries de samedi soir,

C'est pas parce que c'est drôle qu'on rit,

Les fiancés de l'armoire à linge,

Comme un photo-roman d'amour, Cœur de papa,

Monsieur Ribadier's système, Nurse Jane goes to Hawaï,

La baby sitter, Appelez-moi Stéphane,

Essayez donc nos pédalos, Mousse, Ça s'peut pas,

Déridez-vous, Une farce de la nature,

Le bonheur c'est pas bon pour la santé, 
Attention au bouton, Du sang bleu dans les veines, Les dernières chaleurs,

Transport en commun, Boeing Boeing, Jello aux fraises pas de fraise, Pepsie,

Caviar ou lentilles? Poivre et sel,

Chez Paul-ette, bière, vin, liqueur et nouveautés,

Le tout pour le tout,

Beaux bébés de banlieue, voisine de cuisine,

Clarisse, Alexandre, Virgile et tous les autres,

Les Moineau chez les Pinson,

Meurtre à l'Auberge des Gouverneurs, Tu et nous, tuez-nous,

Au revoir Charlie, Adieu les Olympiques ${ }^{2}$.

Il va sans dire que Le roi boiteux ne changeait pas grand chose à l'inflation ni au chômage. Il présentait du moins l'intérêt d'en rire d'un autre rire, de nous distraire des journées pluvieuses sans nous distraire de préoccupations plus fondamentales. Tout un programme en effet que celui offert par le Nouveau Théâtre Expérimental de Montréal ${ }^{3}$. De cette “épopée sanglante et grotesque en six pièces et un épilogue», les spectateurs de l'École Nationale de Théâtre ont pu voir les trois premiers épisodes, et ce d'une façon assez originale pour qu'on s'y arrête un peu. Chacun d'eux constitué par une pièce en soi, était offert au rythme du théâtrefeuilleton comme suit :

$\begin{array}{ll}\text { lundi et jeudi : } & \text { La naissance } \\ \text { mardi et vendredi : } & \text { L'Enfance } \\ \text { mercredi et samedi : } & \text { Le Printemps }\end{array}$

Ce qui laissait à chacun assez de liberté pour organiser ses soirées en évitant de s'infliger la sur-dose de cinq heures d'affilée (à noter que bon nombre de spectacles expérimentaux échoue souvent pour mal évaluer la capacité d'endurance de leurs victimes). L'expérience fut concluante si on en juge par l'affluence du public, une demi-heure en avance sur la plupart des représentations. Et pour ceux qui, comme moi, préféraient tout apprécier d'un coup, on offrait un samedi les trois pièces (avec un battement nécessaire) entre $14 \mathrm{~h} 30$ à 22 heures. Là-encore, l'épreuve du marathon théâtral (bien connu chez les Anciens), était adoucie par l'accueil réservé au public entre chaque performance : collation, repas, boissons, préparés et offerts par la troupe qui reprenait ainsi régulièrement contact avec le spectateur. Inutile d'insister sur la chaleur des rapports, les échanges entre salle et scène et le type de jeu en découlant (quand on compare à l'arrivée dans les granges estivales des autobus nolisés qui déversent leur pesant de spectateurs au guichet, pour les reprendre après les consommations...). Fin de la parenthèse sur les conditions de réception du spectacle de Jean-Pierre Ronfard.

Vie et mort du Roi boiteux, c'est aussi une expérience originale au niveau de la mise en scène. Autant que faire se pouvait, les spectacles étaient donnés en plein air, dans la verdure de la cour abondamment 
fleurie pour l'occasion, de l'École Nationale de Théâtre 4 . Utilisation des décors naturels (arbres, bâtiments environnants, échelles de secours) et proximité des spectateurs disséminés sur des gradins dans la cour. Une scénographie de rechange était prête en cas d'orage. C'est celle, moins séduisante mais tout aussi ingénieuse, que j'ai pu apprécier dans le gymnase de l'École. Peut-être la froideur et la rectitude des cloisons flanquées d'échelles murales, le haut plafond d'où pendaient filets, trapèzes et cordes lisses, contribuaient-ils par contraste à souligner le dynamisme et la fantaisie du jeu theâtral. L'austérité des lieux était d'ailleurs pondérée, par d'immenses toiles masquant latéralement les "coulisses" et, de face, l'aire de dégagement des comédiens. Sur ces toiles, un arbre généalogique des personnages et de grands planisphères à l'ancienne qui éclatent l'action entre le quartier montréalais de l'Arsenal et les quatre continents. Télescopage des graphies et des toponymies: Abitibi, Abyssinie, Amazonie se relancent l'une l'autre, croisant le son avec Azerbajan, Matapédia, Alaska, Panama et d'autres : Kilimanjaro, Acapulco, Titicaca, Gondwana, sans oublier les barbares Krasnoiask et Irkoutsh... Une telle débauche sonore et géographique n'a pas pour seule fonction l'exotisme facile et le dépaysement a bon compte. Elle est inscrite dans la trame dramatique : chaque personnage, chaque enfant des ruelles, chacun(e) dans l'Est est persuadé(e) d'être roi ou reine d'une illustre lignée, qui Premier (François de son prénom!), Augustine (Labelle de son nom, morte en couches), Catherine (dite la Reine-Mère), le vieux Père Roberge (Prince d'Abitibi), le p'tit Moïse (abandonne par Judith Roberge dans une corbeille "Au pied du Courant" !), chacun(e) cohabitant avec des Roy Williams, Sandy Park, Marc Lemieux et Freddy Dubois. C'est la saga des Roberge et des Ragone (prononcer à l'italienne), familles rivales aux cruels appétits. On aura reconnu, sur le mode parodique, les références shakespeariennes ${ }^{5}$, mais aussi avec Leila, Lou Birkanian et le Caucase, les clins d'œil aux contes et mythologies perses, arméniens et gréco-latins ${ }^{6}$, et plus généralement l'imagerie populaire des contes d'enfants?.

En fait, tout semble partir des jeux de ruelles de deux "gangs" d'adolescents. Selon une technique du point de vue déjà fort utilisée dans le roman, Ronfard focalise (et fait singer) les ambitions et mesquineries des "grands" par les "petits", leur spontanéité et leur imagination. Ceux-ci investissent les travers de ceux-là en les magnifiant jusqu'à l'épique, mais un épique tourné en dérision. Car tout se passe sous l'œil goguenard du patriarche Fillipo Ragone, dit Le Débile. Omniprésent, "effoiré" dans sa chaise de handicapé, tordu de grimaces, l'aïeul n'est qu'un simulateur. II se réfugie dans un mutisme entrecoupé de gueulantes pour mieux observer son entourage (et nous inviter à en faire autant) : c'est lui qui inscrit la distance dans le jeu. Seul Richard, le héros boîteux, aura droit à ses confidences (et dans une certaine mesure, à son aide, à son savoir). Le spectateur est ainsi placé en porte-à-faux entre trois discours : celui des adultes, à l'emphase parfois "maghanee" (on pense au fameux Cid ducharmien); le babil enfiévré des enfants (dont le comportement l'emporte parfois en maturité sur celui des parents - là encore, voir Ducharme); enfin, 
silencieux mais éloquent, le discours du vieillard. Ce dernier sémantise les deux autres sur le mode parodique. Impossible de prendre au sérieux les prétentions des uns ni les élucubrations des autres, qui toutes deux se dénonçaient déjà mutuellement. De sorte que le texte de Ronfard (texte compris au sens de trace écrite et de représentation scénique) réussit la gageure de jouer constamment sur les trois tableaux. L'épaisseur sémantique ainsi produite, confère aux nombreux effets comiques une toute autre densité, générant un rire en tout point différent de celui des farces estivales évoquées plus haut.

Non que Le Roi boitteux nous en impose par une structure dramatique pesante et prétentieuse. Le poids du sens lui-même n'écrase jamais cette production dynamique (le seul rythme du jeu l'en empêcherait) ${ }^{8}$. Au contraire, les niveaux de sens se bousculent et se relancent indéfiniment avec un dynamisme que ne démentent jamais la juxtaposition des tableaux et des lieux, et la progression parallèle des intrigues. "Jean-Pierre Ronfard réinvente la Fête, retrouve l'esprit et le souffle des mystères médiévaux, des "pageants", le foisonnement des carnavals", lit-on dans la préface à l'édition Leméac. Le tout avec un sens aigü du suspens puisé dans la tradition feuilletonnesque: ce n'est pas un hasard si VLB se trouve brocardé au détour de telle réplique de Richard Premier : «On est ben amôché, race de monde!». En somme un merveilleux spectacle d'été dont on espère connaître la fin à la saison prochaine et sur lequel les amateurs du théâtre écrit pourront revenir à loisir.

1. Cf. la saison d'été 1977 dans le Jour hebdo.

2. Liste authentique! Référence : Le Devoir du 8 août $1981 \ldots$ On pourrait simplement rajouter: le texte intégral de l'Évangile selon St-Marc, à l'Église StSauveur, le même mois.

3. Vie et mort du Roi boiteux, une épopée sanglante et grotesque (en six pièces et un épilogue) de Jean-Pierre Ronfard, T. 1. et T. 2. Théâtre/Leméac, avec une préface de Jean-Cléo Godin et Pierre Lavoie.

4. L'École Nationale de Théâtre offrait ses murs au Nouveau Théâtre Expérimental, dans l'attente de leur nouveau local, l'Espace libre (après la cession - et la scission - de la Maison Beaujeu, occupée maintenant par le Théâtre Expérimental des Femmes).

5. Roméo et Juliette, mais aussi le Songe à travers l'évocation de I'Halloween, et tous les drames historiques revus et corriges dans ces "Shakespeare Follies "...

6. Le Minotaure se trouve garder la Passe des Rocheuses, alors que François Premier qui cite ses sources ("J'ai fait mon cours classique, Sacrament!"), s'exclame à l'agonie: "Héraclès, Sisyphe, Prométhée, mes compagnons, Calvaire! "...

7. Imagerie mâtinée du discours mal assimilé des mass-media : "...au milieu des esclaves circasiennes, les poches bourrées de pétro-dollars"...

8. La portée philosophique et socio-culturelle de l'œuvre est abordée avec plus de détails dans l'excellente préface à l'édition Leméac. Rappelons simplement le prière d'insérer: "Au sein du pouvoir absolu de l'homme, le doute sur sa légitimité et l'image de sa faiblesse... Sur le théâtre du monde, une horde de personnages qu'agitent des passions contradictoires, qui se prennent tous pour des reines et des rois, mais qui boitent. L'image d'un peuple en perpétuelle évolution et qui ressemble à n'importe quel peuple de la terre". 